\title{
INTERSECTIONS OF TANGENT CONVEX CURVES
}

\author{
TUDOR ZAMFIRESCU \\ (Received 12 November 1980) \\ Communicated by $\mathbf{H}$. Lausch
}

\begin{abstract}
We prove in this note that if two convex curves are internally tangent flie on the same side of the common tangent), then in most cases they also cut each other infinitely many times. It follows that, in a certain sense, if two convex curves have an odd number of common points, then in general they are externally tangent (lie on different sides of the common tangent). The sense of the expressions most cases and in general remains to be precised.
\end{abstract}

1980 Mathematics subject classification (Amer. Math. Soc.): 52 A 10.

Let $\mathcal{C}$ be the space of all (always closed and smooth) convex curves in the plane. Endowed with the Hausdorff metric $d, \mathcal{C}$ is a Baire space. This result goes essentially back to Klee [2] and Gruber [1]. It is only routine to check that the spaces $\mathscr{T}_{i}, \mathcal{T}_{e} \subset \mathcal{C}^{2}$ of all pairs of internally, respectively externally tangent curves in $\mathcal{C}$ are Baire spaces too. Thus, the union $\mathcal{T}_{\text {of }} \mathcal{T}_{i}$ with $\mathcal{T}_{e}$ is, with the topology induced by $\mathfrak{C}^{2}$, a Baire space.

Let $\mathcal{O}$ be the subspace of $\mathcal{T}$ consisting of all pairs of curves in $\mathcal{C}$ having an odd number of common points and let $\mathscr{F}$ be the set of all pairs in $\mathscr{T}_{i}$ of curves having finitely many common points.

THEOREM 1. In the space $\mathcal{T}_{i}$ of all pairs of internally tangent (closed, smooth) convex curves, the subspace $\mathcal{T}^{*}$ of all pairs of curves having only one tangent point and cutting each other in every neighbourhood of the tangent point is residual.

Before giving a proof, let us make some remarks. From Theorem 1 it follows that $\mathscr{F}$ is of first Baire category in $\mathscr{T}_{i}$. In this sense (always) we say that most

OCopyright Australian Mathematical Society 1981 
pairs of internally tangent convex curves do not belong to $\mathscr{F} . \mathcal{O}$ is not a Baire space, but since $\mathscr{F}$ is of first Baire category in $\mathcal{T}$ and

$$
\sigma_{e} \subset \theta \subset \sigma_{e} \cup \mathscr{F}
$$

it follows that $\mathcal{\theta}$ is of second, while $\theta-\sigma_{e}$ of first Baire category in $\mathcal{T}$. In this sense we claimed that, in general, pairs of $\theta$ lie in $\mathcal{T}_{e}$.

Proof of Theorem 1. Let $\mathscr{F}_{n}$ be the set of all pairs in $\mathscr{T}_{i}$ having no cutting point at distance at most $n^{-1}$ or having some tangent point at distance at least $n^{-1}$ from any point of tangency. We show that $\mathscr{F}_{n}$ is nowhere dense in $\mathcal{T}_{i}$.

Let $Q$ be an open set in $\mathscr{T}_{i}$ and $\left(C_{1}, C_{2}\right) \in \mathbb{Q}$. Let $\varepsilon>0$ be such that $\left(C_{1}^{\prime}, C_{2}^{\prime}\right) \in \mathbb{Q}$ whenever $d\left(C_{j}^{\prime}, C_{j}\right)<\varepsilon(j=1,2)$ and $\left(C_{1}^{\prime}, C_{2}^{\prime}\right) \in \mathcal{T}_{i}$. Also, let $x$ be a tangent point of $C_{1}$ and $C_{2}$ and let $T$ be one of the half-lines determined by $\boldsymbol{x}$ on the common tangent line at $x$. Obviously, there exist polygons $\boldsymbol{P}_{1}, \boldsymbol{P}_{2}$ with $x$ as vertex, with no edge on $T$, at distance less than $\varepsilon$ from $C_{1}, C_{2}$ respectively and such that every common point of $P_{1}, P_{2}$ different from $x$ is not a vertex of any of them, but a cutting point of two edges. Let $y_{j}$ be the vertex of $P_{j}$ such that $x y_{j}$ is an edge of $P_{j}$ and $y_{1}, y_{2}, T$ lie on the same side of the line orthogonal at $x$ to $T$. Let $\alpha_{j}$ be the angle between $x y_{j}$ and $T$, and suppose, without loss of generality, that $\alpha_{1} \leqslant \alpha_{2}$. Let $z_{2}$ be the vertex of $P_{2}$ following $y_{2}$. The line through $y_{2}, z_{2}$ intersects $T$ (if $\varepsilon$ is small enough) at some point $u$. If $y$ converges to $x$ on $T$, then the distance $\delta(y)$ between $x$ and the intersection point of $y y_{2}$ with the line through $x, y_{1}$ converges to zero. Thus, we can find a point $y_{0} \neq x$ on $T$ at distance less than $\varepsilon$ from $x$, closer to $x$ than $u$ and such that $\delta\left(y_{0}\right)<(3 n)^{-1}$ and $y_{0} y_{2}$ meets $x y_{1}$ in a point $t \neq y_{1}$. Let

$$
\begin{aligned}
& P_{1}^{\prime}=P_{1}, \\
& P_{2}^{\prime}=\left(P_{2}-x y_{2}\right) \cup x y_{0} \cup y_{0} y_{2} .
\end{aligned}
$$

Clearly, $d\left(C_{j}, P_{j}^{\prime}\right)<\varepsilon(j=1,2)$.

Now, there exists $\nu>0$ such that, for any pair $\left(C_{1}^{\prime}, C_{2}^{\prime}\right) \in \mathfrak{T}_{i}$ with $d\left(C_{j}^{\prime}, P_{j}^{\prime}\right)<$ $\nu$, the following holds:

(i) $\left(C_{1}^{\prime}, C_{2}^{\prime}\right) \in \mathbb{Q}$,

(ii) the tangent points of $C_{1}^{\prime}, C_{2}^{\prime}$ are necessarily at distance at most $(3 n)^{-1}$ from $x$,

(iii) to each cutting point $c$ of $\boldsymbol{P}_{1}^{\prime}, \boldsymbol{P}_{2}^{\prime}$ it corresponds precisely one cutting point of $C_{1}^{\prime}, C_{2}^{\prime}$ at distance at most $(3 n)^{-1}$ from $c$.

It follows that, for every such pair $\left(C_{1}^{\prime}, C_{2}^{\prime}\right)$,

$1^{\circ}$. no tangent points have distance more than $2(3 n)^{-1}$ from each other.

$2^{\circ}$. the distance between the cutting point $t^{\prime}$ of $C_{1}^{\prime}, C_{2}^{\prime}$ corresponding to $t$ and any tangent point $x^{\prime}$ of $C_{1}^{\prime}, C_{2}^{\prime}$ is at most equal to the distance from $t$ to $t^{\prime}$ plus the distance $\delta\left(y_{0}\right)$ from $t$ to $x$ plus the distance from $x$ to $x^{\prime}$, hence at most $n^{-1}$. 
Therefore $\mathscr{F}_{n}$ is nowhere dense in $\mathscr{T}_{i}$. Thus, the set $\mathscr{T}_{i}-\mathscr{F}^{*}=\cup_{n-1}^{\infty} \mathscr{F}_{n}$ is residual in $\mathscr{T}_{i}$.

Without any additional difficulty the following $n$-dimensional version can be proved.

THEOREM 2. In the Baire space of all pairs of internally tangent closed smooth convex surfaces, the subspace of all pairs of surfaces having only one tangent point and cutting each other in every neighbourhood of the tangent point is residual.

Let $\delta$ be the subspace of $\mathscr{T}_{i}$ of all pairs of internally tangent convex curves, one of which is a circle. It is easily seen that $\mathcal{S}$ is a Baire space too. The following result is analogous to Theorem 1; the proof is similar and therefore omitted.

THEOREM 3. $\mathscr{F}^{*} \cap \mathcal{S}$ is residual in $\mathcal{S}$.

A special interest of this theorem lies in the following apparent contradiction: On one hand, for most pairs in $\mathcal{S}$, the (unique) tangent point is not isolated in the set of all common points. On the other hand, by Theorem 2 in [3], most curves in $\mathcal{C}$ have vanishing curvature a.e. Any circle tangent to such a curve at a point where the curvature vanishes does not meet again the curve in some neighbourhood of that point. Thus, for most convex curves in $\mathcal{C}$ and almost all tangent circles (in an obvious measure-theoretical sense), there is a tangent point isolated in the set of all common points!

\section{References}

[1] P. M. Gruber, 'Die meisten konvexen Körper sind glatt, aber nicht zu glatt', Math. Ann. 229 (1977), 259-266.

[2] V. Klee, 'Some new results on smoothness and rotundity in normed linear spaces', Math. Ann. 139 (1959), 51-63.

[3] T. Zamfirescu, 'The curvature of most convex surfaces vanishes almost everywhere', Math. Z. 174 (1980), 135-139.
Abteilung Mathematik
Universität Dortmund
D-4600 Dortmund 50, Postfach 500500
Federal Republic of Germany 